\title{
2'-O-Methyl RNA/Ethylene-Bridged Nucleic Acid Chimera Antisense Oligonucleotides to Induce Dystrophin Exon 45 Skipping
}

\author{
Tomoko Lee ${ }^{1}$, Hiroyuki Awano ${ }^{2}$, Mariko Yagi ${ }^{3}$, Masaaki Matsumoto ${ }^{2}$, Nobuaki Watanabe ${ }^{4}$, \\ Ryoya Goda ${ }^{4}$, Makoto Koizumi ${ }^{5}$, Yasuhiro Takeshima ${ }^{1}$ and Masafumi Matsuo ${ }^{6, *}$ \\ 1 Department of Pediatrics, Hyogo College of Medicine, Nishinomiya 6638501, Japan; \\ leeleetomo@me.com (T.L.); ytake@hyo-med.ac.jp (Y.T.) \\ 2 Department of Pediatrics, Kobe University Graduate School of Medicine, Kobe 6500017, Japan; \\ awahiro@med.kobe-u.ac.jp (H.A.); mmatsu@med.kobe-u.ac.jp (M.M.) \\ 3 Nikoniko House Medical and Welfare Center, Kobe 6511102, Japan; marikoyagi.sgt@gmail.com \\ 4 Drug Metabolism \& Pharmacokinetics Research Laboratories, Daiichi Sankyo Co., Ltd., Tokyo 1408710, \\ Japan; watanabe.nobuaki.nc@daiichisankyo.co.jp (N.W.); goda.ryoya.u4@daiichisankyo.co.jp (R.G.) \\ 5 Modality Research Laboratories, Daiichi Sankyo Co., Ltd., Tokyo 1408710, Japan; \\ koizumi.makoto.h7@daiichisankyo.co.jp \\ 6 Department of Physical Therapy, Faculty of Rehabilitation, Kobe Gakuin University, Kobe 6512180, Japan \\ * Correspondence: mmatsuo@reha.kobegakuin.ac.jp; Tel./Fax: +81-78-974-6194
}

Academic Editors: Susan Fletcher and Steve Wilton

Received: 13 December 2016; Accepted: 7 February 2017; Published: 10 February 2017

\begin{abstract}
Duchenne muscular dystrophy (DMD) is a fatal muscle-wasting disease characterized by dystrophin deficiency from mutations in the dystrophin gene. Antisense oligonucleotide (AO)-mediated exon skipping targets restoration of the dystrophin reading frame to allow production of an internally deleted dystrophin protein with functional benefit for DMD patients who have out-of-frame deletions. After accelerated US approval of eteplirsen (Exondys 51), which targets dystrophin exon 51 for skipping, efforts are now focused on targeting other exons. For improved clinical benefits, this strategy requires more studies of the delivery method and modification of nucleic acids. We studied a nucleotide with a $2^{\prime}-O, 4^{\prime}$-C-ethylene-bridged nucleic acid (ENA), which shows high nuclease resistance and high affinity for complementary RNA strands. Here, we describe the process of developing a $2^{\prime}-O$-methyl RNA $\left(2^{\prime}-\mathrm{OMeRNA}\right) / \mathrm{ENA}$ chimera AO to induce dystrophin exon 45 skipping. One 18-mer 2'-OMeRNA/ENA chimera (AO85) had the most potent activity for inducing exon 45 skipping in cultured myotubes. AO85 was administered to $m d x$ mice without significant side effects. AO85 transfection into cultured myotubes from $13 \mathrm{DMD}$ patients induced exon 45 skipping in all samples at different levels and dystrophin expression in 11 patients. These results suggest the possible efficacy of $\mathrm{AO}$-mediated exon skipping changes in individual patients and highlight the 2'-OMeRNA/ENA chimera $\mathrm{AO}$ as a potential fundamental treatment for DMD.
\end{abstract}

Keywords: 2'-O,4'-C-ethylene-bridged nucleic acid; ENA; antisense oligonucleotide; exon skipping therapy; Duchenne muscular dystrophy

\section{Introduction}

Duchenne muscular dystrophy (DMD) (OMIM 310200) is the most common inherited muscle disease in childhood, affecting more than one in every 3500 live-born boys. DMD is caused by mutations in the dystrophin gene and characterized by dystrophin deficiency in muscles. It is a progressive muscle-wasting disease and usually fatal in the third or fourth decades of life. Although general medical treatments and physical therapy have slowly increased life expectancy [1,2], 
establishment of a fundamental treatment that leads to dystrophin expression has long been the ultimate goal in DMD research.

To recover dystrophin expression in DMD, gene replacement therapy is a logically appropriate strategy, but more studies are needed to form the basis for clinical translation [3,4]. As an alternative, induction of exon skipping with antisense oligonucleotides (AOs) that convert out-of-frame dystrophin mRNA into in-frame sequences has been proposed [5]. Exonic deletion mutations in the dystrophin gene cause not only DMD but also Becker muscular dystrophy (BMD), a milder progressive muscle-wasting disease. A translational reading frame rule explains the difference between DMD and BMD [6]: Out-of-frame exon deletion mutations create premature stop codons that result in the more severe DMD phenotype because of a total lack of the dystrophin protein. In contrast, exon deletion mutations that maintain the original reading frame in the mRNA lead to the milder BMD phenotype because a mutated, but still functional, dystrophin protein can be expressed from the mRNA. Therefore, exon skipping allowing one exon to be omitted from the mutated dystrophin mRNA can be used to restore the reading frame and produce the internally deleted dystrophin [7]. Compared with gene replacement therapy, the benefits of exon skipping with AOs include the ability to use the endogenous gene and straightforward chemical synthesis of AOs.

We demonstrated the first clinical study of exon skipping therapy using a phosphorothioateoligonucleotide against dystrophin exon 19 and showed successful induction of exon skipping and dystrophin expression in skeletal muscle in a DMD patient (Patient 0) [8]. However, the effectiveness was not enough to improve his motor function. This study has demonstrated a proof of concept as an AO-mediated exon skipping to treat DMD [9]. Subsequently, in order to develop successful AO-mediated exon skipping therapy, various kinds of modified AOs have been chemically synthesized, with some of the most prominent being phosphorodiamidate morpholino oligonucleotides (PMOs), 2'-O-methyl (2'-OMe) AOs, locked nucleic acid (LNA), peptide nucleic acid (PNA), and tricycle-DNA (tcDNA) [10,11]. Furthermore, chimera AOs such as anhydrohexitol nucleic acid (HNA)/2'-OMe phosphorothioate (2'OMePS), cyclohexenyl nucleic acid (CeNA)/2'OMePS, altritol nucleic acid (ANA) $/ 2^{\prime} \mathrm{OMePS}$, and morpholino nucleic acid (MNA)/2'OMePS have been investigated $[12,13]$. Among these AOs, two AOs comprising different monomers-PMOs [14] and 2'-OMePS [15]—-were developed to induce skipping of dystrophin exon 51 and have undergone clinical trials. These trials yielded good results, enabling improvements in ambulation in DMD patients [16-19]. One PMO oligonucleotide, eteplirsen-mediated (Sarepta Therapeutics Inc., Cambridge, MA, USA) exon skipping therapy led to a slower rate of decline in ambulation compared to historical controls [18]. After a long heated discussion [20], eteplirsen has received accelerated approval from the US Food and Drug Administration (FDA) [21]. At the same time FDA stated that a clinical benefit of eteplirsen has not been established [22]. There is controversy as to whether it is really beneficial [23-25].

Now, AO-mediated exon skipping therapy is reaching the final stage to provide a treatment for DMD patients. Efforts are focused on targeting other exons $[4,26]$. However, it is true that this therapy can stand further improvement in modification of nucleic acids, their delivery method, and evaluation methods [10]. To improve clinical benefits, more studies are needed.

After establishing the proof of concept as AO-mediated exon skipping therapy, we have continuously developed AOs comprising a modified nucleotide with an ethylene bridge between oxygen at the $2^{\prime}$-position and carbon at the $4^{\prime}$-position of ribose $\left(2^{\prime}-O, 4^{\prime}-C\right.$-ethylene-bridged nucleic acid; ENA ${ }^{\circledR}$, Daiichi Sankyo Co., Ltd., Tokyo, Japan), which is highly nuclease resistant and shows a high affinity for complementary RNA strands [27]. Here, we describe the progress in developing the 2'-O-methyl RNA(2'-OMeRNA)/ENA chimera AO to induce dystrophin exon 45 skipping. 


\section{Identification of AO for Exon 45 Skipping}

\subsection{A Modified Nucleic Acid of ENA ${ }^{\circledR}$}

To improve the efficacy of nucleic acids, several modified nucleic acids have been created to provide higher nuclease resistance and high affinity for complementary sequences. ENA with an ethylene bridge between the oxygen and carbon of ribose (Figure 1) is thermodynamically stable and highly nuclease resistant [28,29] and has a high affinity for complementary RNA strands [30,31]. In fact, the ability of an AO consisting of the 2'-OMeRNA/ENA chimera to induce exon 19 skipping is more than 40 times stronger than that of the conventional phosphorothioate backbone oligonucleotides, making it a promising candidate for use as a low-toxicity, high-affinity oligonucleotide in the long-term treatment of DMD [32]. Furthermore, a 2'-OMeRNA/ENA chimera against dystrophin exon 41 encoding a nonsense mutation has been shown to induce efficient skipping of the mutated exon 41 [33]. Therefore, we decided to use 2'-OMeRNA/ENA chimera oligonucleotides for exon skipping.

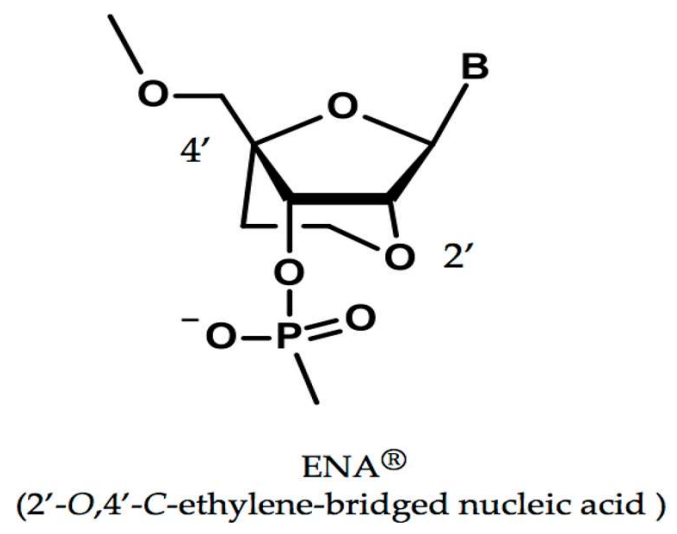

Figure 1. Structure of $2^{\prime}-O, 4^{\prime}-C$-ethylene-bridged nucleic acid (ENA). ENA has an ethylene bridge between the oxygen at the $2^{\prime}$-position and carbon at the $4^{\prime}$-position of ribose. $\mathrm{B}$ indicates base.

\subsection{2'-OMeRNA/ENA Chimera AO to Skip Exon 45}

Exon skipping therapy is mutation specific. To develop the broad therapeutic applicability of exon skipping therapy, we focused on induction of exon 45 skipping because it can be applied for treatment in approximately $9 \%$ of DMD patients $[19,34]$. The best AO to induce exon 45 skipping was identified by a trial-and-error procedure as described previously [35]. 2'-OMeRNA/ENA chimera AOs consisting of $2^{\prime}-O$-methyl RNA with phosphodiester backbone, and ENA residues (at cytosines and thymines or at both the $5^{\prime}$ and $3^{\prime}$-ends) were employed. As the first screening for the identification of AOs against exon 45, five 15-mer AOs (AO32-36) to cover the splicing enhancer sequence of exon 45 were designed (Figure 2a) and examined for their ability to induce exon 45 skipping. Among five AOs, only one, AO33, induced exon 45 skipping (Figure 2b). To identify a more suitable AO, another set of 18-mer AOs (AO85-87) neighboring AO33 was designed (Figure 2a). Although all three AOs induced exon 45 skipping, only AO85 did so perfectly (Figure 2c). Therefore, AO85 was selected as the optimal $\mathrm{AO}$ for exon 45 skipping. 
a)

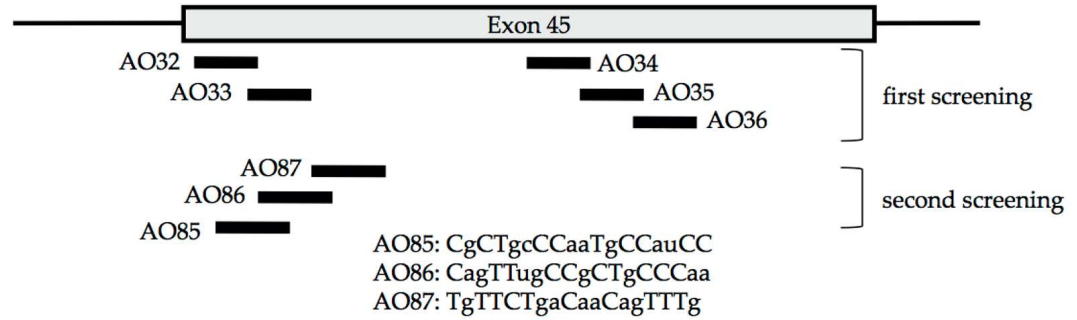

b)

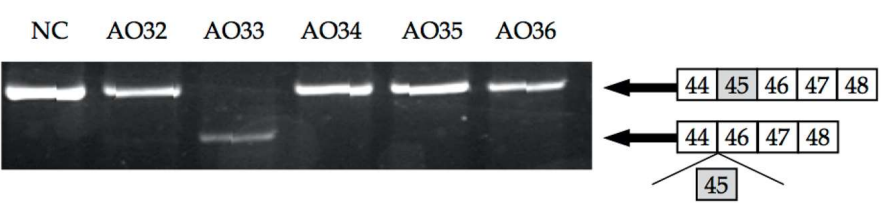

c)

$\begin{array}{lllll}\mathrm{NC} & \mathrm{AO} 33 & \mathrm{AO} 85 & \mathrm{AO} 86 & \mathrm{AO} 87\end{array}$

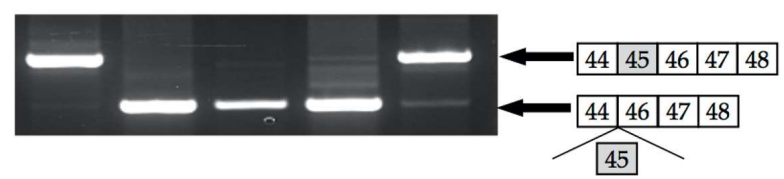

Figure 2. Identification of the best antisense oligonucleotide (AO) to induce exon 45 skipping. 2'-OMeRNA/ENA chimera AOs were designed to cover splicing enhancers in exon 45 and synthesized. A portion of $200 \mathrm{nM}$ of each $\mathrm{AO}$ was transfected into myotubes. The resulting dystrophin mRNA was analyzed by RT-PCR amplification. The procedure of this analysis has been described in detail by Takeshima et al. [34]. (a) Locations of AOs are schematically described. Box and bars indicate exon 45 and flanking introns, respectively. Bars represent AOs. The AOs shown in the upper and the lower halves were used in the first and second screening steps, respectively. Nucleotide sequences of AO85, AO86, and AO87 were described (bottom). Upper and lower-case letters represent ENA-modified residues and 2'-O-methyl RNA, respectively; (b) Electropherograms of RT-PCR products. A dystrophin cDNA fragment from exons 44 to 48 was PCR amplified. PCR amplification revealed two products in AO33-treated cells (AO33) and one product in other treatments (AO32, AO34, AO35, and AO36). The additional smaller product in AO33-treated cells lacked exon 45, indicating exon 45 skipping. Lane NC represents non-transfected control (NC). The exons in the amplified products are shown schematically on the right, and the shaded box represents the AO target exon; (c) Electropherograms of RT-PCR amplification products of the second screening step for AO-induced dystrophin exon 45 skipping. All three AOs induced exon 45 skipping, but only AO85 induced exon 45 skipping perfectly.

\subsection{Characterization of $A O 85$}

The half-maximum effective concentration of AO85 was determined in a cell-free splicing system and found to be $58 \mathrm{nM}$ [36]. To determine the stability of AO85, it was incubated with human and mouse blood. The mass peak corresponding specifically to the unchanged form of the full-length AO85 was monitored by LC-MS/MS system. The peak area ratio of AO85 to the internal standard (IS) remained stable for 2 and $8 \mathrm{~h}$ in human and mouse blood, respectively (Figure 3), demonstrating that AO85 remained as the full-length unchanged form over the time course of the incubation in human and mouse blood. Consistent with previous studies [28,29] on other ENA-AOs, AO85 was found to be stable in blood.

In our previous reports AO85 at $400 \mathrm{nM}$ caused a 30\% decrease in cell viability in MTT assay using fibroblasts [37]. To examine the toxicity of AO85, we performed a single dose-escalation study using $m d x$ mice, an animal model of DMD (Table 1). The $m d x$ mice received intravenous administration of AO85 at doses of $0,5,15$, and $50 \mathrm{mg} / \mathrm{kg}$. They were observed for 14 days, and no abnormalities, including in body weight change, were found. Autopsy examination at Day 14 revealed no apparent abnormal pathological change in $m d x$ mice treated with 0,5 , and $15 \mathrm{mg} / \mathrm{kg}$. However, four of five mice treated with $50 \mathrm{mg} / \mathrm{kg}$ of AO85 showed renal enlargement, and two of five showed cysts on the renal surface. These findings suggested renal damage caused by high-dose AO85. 
a) Stability in human blood

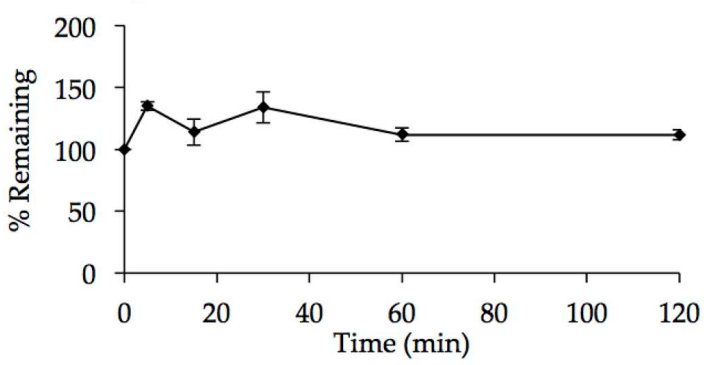

b) Stability in mouse blood

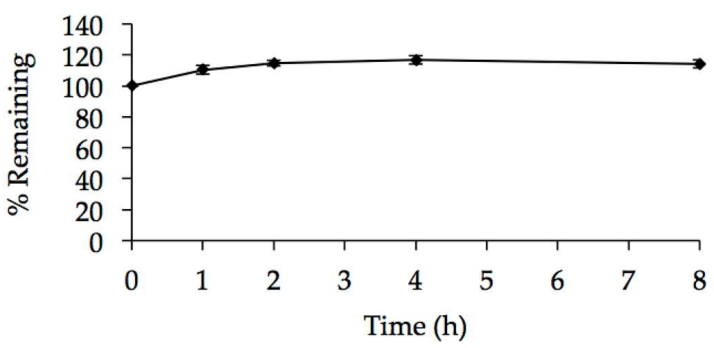

Figure 3. Stability of AO85 in human and mouse blood. Human blood was collected from three healthy volunteers, who had received no drugs for more than one week before blood sampling, with written informed consent. A $5 \mu \mathrm{L}$ aliquot of AO85 solution $(1.0 \mathrm{mg} / \mathrm{mL})$ was added to the freshly obtained human individual blood ( $2.5 \mathrm{~mL}$ each, $n=3)$ at a final concentration of $2.0 \mu \mathrm{g} / \mathrm{mL}$. Aliquots of the blood incubation mixture were taken at the time points of $0,5,15,30,60$, and $120 \mathrm{~min}$ after incubation at $37^{\circ} \mathrm{C}$. Mouse blood was obtained from male mice (C57BL/6NCrlCrlj, $n=42$, Charles River Laboratories, Yokohama, Japan) under isoflurane anesthesia. A $20 \mu \mathrm{L}$ aliquot of AO85 solution $(1.0 \mathrm{mg} / \mathrm{mL})$ was added to the freshly obtained pooled mouse blood $(10 \mathrm{~mL})$ at a final concentration of $2.0 \mu \mathrm{g} / \mathrm{mL}$, which was divided into three portions prior to incubation. Aliquots of the blood incubation mixture were taken at the time points of $0,1,2,4$, and $8 \mathrm{~h}$ after incubation at $37^{\circ} \mathrm{C}$. An aliquot $(100 \mu \mathrm{L})$ of each blood sample following centrifugation at $15,000 \mathrm{rpm}$ at $4{ }^{\circ} \mathrm{C}$ for $5 \mathrm{~min}$ was mixed with $100 \mu \mathrm{L}$ aqueous methanol containing an internal standard (IS) compound, followed by filtration using the Amicon Ultra filter (YM-50) and concentration using the Amicon Ultra filter device (YM-3). The samples were analyzed by LC-MS/MS system (HPLC: Ultimate, MS: TSQ Vantage, Thermo Fisher Scientific Inc., Waltham, MA, USA), and peak area ratio (AO85/IS) was determined. Then, the data at each time point were expressed as stability \%, with peak area ratio at $0 \mathrm{~min}$ being $100 \%$. (a) Stability is shown as \% remaining at each time point. In human blood, the peak area ratio of AO85/IS remained stable even for $120 \mathrm{~min}$; (b) In mouse blood, the peak area ratio of AO85/IS remained stable even for $8 \mathrm{~h}$.

Table 1. Dose escalation administration of AO85 in $m d x$ mice.

\begin{tabular}{cccc}
\hline AO85 (mg/kg) & Number of Deaths (Case) & General Condition & Pathological Findings (at Day 14) \\
\hline 0 & 0 & Good & Normal \\
5 & 0 & Good & Normal \\
15 & 0 & Good & Normal \\
50 & 0 & Good & Renal enlargement $(4 / 5$ cases $)$ \\
& & & Cyst on renal surface $(2 / 5$ cases $)$ \\
\hline
\end{tabular}

Five mice were administered the same concentration of AO85 at four different levels and observed for 14 days.

Next, we performed a repeated-administration toxicity study using $m d x$ mice (Table 2). Intravenous AO85 injection at doses of $0,0.5$, and $5.0 \mathrm{mg} / \mathrm{kg}$ was repeated four times at one-week intervals in five $m d x$ mice. Over four weeks, one mouse receiving $0 \mathrm{mg} / \mathrm{kg}$ died because of varicella; no other animals died. No abnormal finding was observed regarding the general condition, weight change, food intake, blood examination, and pathological findings. This result suggested that repeated administration of AO85 is safe. 
Table 2. Repeated administration of AO85 into $m d x$ mice.

\begin{tabular}{ccccc}
\hline $\begin{array}{c}\text { AO85 } \\
(\mathbf{m g} / \mathbf{k g})\end{array}$ & Deaths & $\begin{array}{c}\text { General } \\
\text { Condition }\end{array}$ & $\begin{array}{c}\text { Laboratory } \\
\text { Examination }\end{array}$ & $\begin{array}{c}\text { Pathological } \\
\text { Findings (at Day 28) }\end{array}$ \\
\hline 0 & 1 & Good & Normal & Normal \\
0.5 & 0 & Good & Normal & Normal \\
5 & 0 & Good & Normal & Normal \\
\hline
\end{tabular}

Four times at one-week intervals, five mice were administered the same concentration of AO85 at three different levels.

\section{Efficacy of AO85 in Myotubes from DMD Patients}

\subsection{AO85 Efficacy in Myotubes from a DMD Patient with a Deletion Mutation of Exon 46-51}

To confirm the dose-dependent effect of AO85, it was transfected into cultured myotubes from a DMD patient with a deletion mutation of exon 46-51. RT-PCR amplification of mRNA in cultured myotubes treated at the concentration over $20 \mathrm{nM}$ revealed a shorter product lacking exon 45 , which was not observed in cultured myotubes at the concentrations lower than $10 \mathrm{nM}$ (Figure 4a). At the concentrations over $100 \mathrm{nM}, 100 \%$ of the dystrophin mRNA showed exon 45 skipping (Figure 4b).

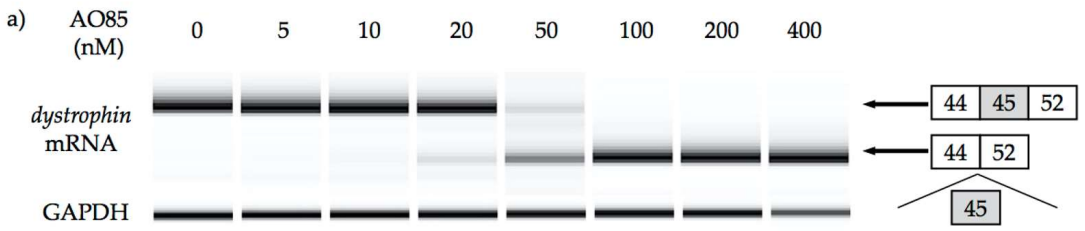

b)

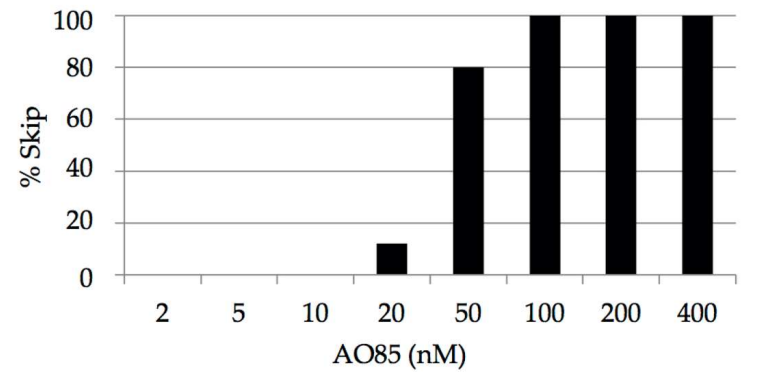

Figure 4. Induction of exon 45 skipping with AO85 in Duchenne muscular dystrophy (DMD) myotubes. Preparation of myotubes from a DMD patient with a deletion mutation of exons 46-51 and examination of exon skipping by AO85 transfection were conducted as described previously [32]. AO85 was added into cultured medium at concentrations of $0,5,10,20,50,100,200$, and $400 \mathrm{nM}$. cDNA was prepared from $1 \mu \mathrm{g}$ of total RNA. The dystrophin mRNA was analyzed by RT-PCR amplification. (a) The result of RT-PCR amplification. At the concentration over $20 \mathrm{nM}$, RT-PCR amplification revealed shorter product lacking exon 45 , which was not observed at the concentration lower than $10 \mathrm{nM}$. Only shorter product was observed at the concentration over $100 \mathrm{nM}$; (b) The exon-45-skipping efficacy by AO85. Skipping efficiencies were determined from gel images by quantifying the skipped products with a DNA 1000 LabChip Kit on an Agilent 2100 Bioanalyzer (Agilent Technologies, Santa Clara, CA, USA). At the concentration over $100 \mathrm{nM}, 100 \%$ of the dystrophin mRNA showed exon 45 skipping.

Next, we analyzed dystrophin expression in cultured myotubes from the same patient with a deletion mutation of exons 46-51 (Figure 5). Before treatment with AO, immunohistochemical staining showed no dystrophin signal. However, at 7 days after transfection with AO85 (50 nM), immunohistochemical staining disclosed antibody-reacting materials in myotubes treated with AO85. 
These results revealed that AO85 transfection could induce exon 45 skipping and lead to dystrophin production in DMD myotubes.

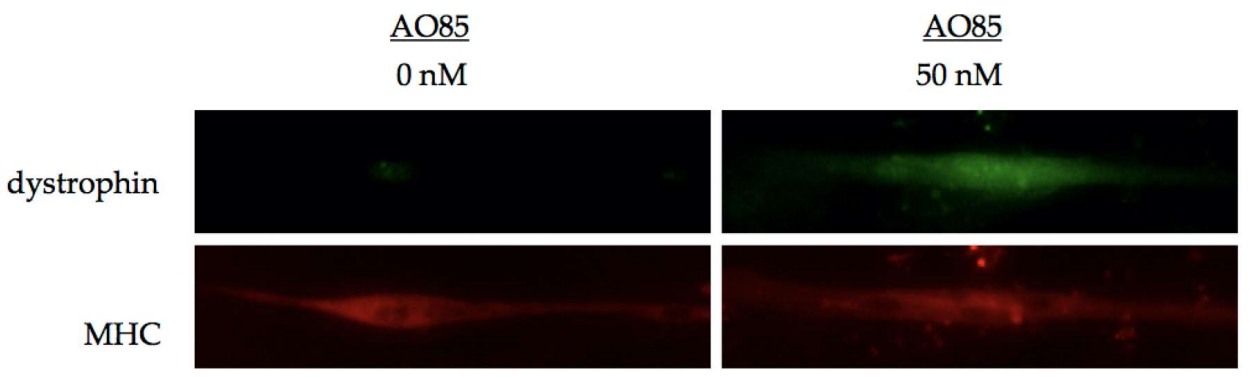

Figure 5. Dystrophin expression in cultured DMD myotubes by AO85 transfection. AO85 was transfected into the myotubes prepared as described in Figure 4 at a concentration of $50 \mathrm{nM}$. Seven days after the transfection, treated myotubes were harvested. After fixation with cold methanol, the fixed myotubes were incubated with polyclonal anti-dystrophin antibody (targeted to the C-terminal region; ab15277, Abcam, Cambridge, UK) and anti-myosin heavy chain (MHC) antibody (sc 101334, Santa Cruz Biotechnology, Dallas, TX, USA). The secondary antibodies were conjugated with Alexa Fluor 488 (Abcam) for dystrophin and Alexa Fluor 568 (Abcam) for the MHC. Immunohistochemical staining of dystrophin and MHC are shown. Before treatment, dystrophin was negative. After the transfection of AO85 (50 nM), myotubes were dystrophin positive, as was MHC staining. A quantitative assessment of dystrophin expression was not performed. Studies on DMD patients in this article were done after obtaining informed consent, in addition to approval from the local ethics committee.

\subsection{AO85 Efficacy in Myotubes from 13 DMD Patients}

We examined the ability of AO85 to induce exon 45 skipping and dystrophin expression in myotubes from 13 DMD patients carrying six different deletion mutations, such as deletions of exon 44 , 46-47, 46-48, 46-49, 46-51, or 46-53. In each case, exon 45 skipping was induced by AO85, as expected; however, the skipping efficiency was different from patient to patient (Table 3). In myotubes from three patients with the exon $46-51$ deletion, more than $80 \%$ of the dystrophin mRNA was exon skipping product while less than $43 \%$ of it was in myotubes carrying the exon $46-47$ deletion. Dystrophin expression was seen in all but two patients. Unexpectedly, in myotubes with the exon 46-51 deletion, dystrophin expression was not apparent even though $82 \%$ showed exon 45 skipping in the mRNA. On the other hand, in myotubes with exon 46-47 deletion, dystrophin expression was apparent in spite of a low rate of exon 45 skipping in the mRNA.

Table 3. AO85-mediated exon 45 skipping and dystrophin expression in 13 DMD patients.

\begin{tabular}{ccccc}
\hline No. & Age & Deleted Exon & Exon-45-Skipped mRNA (\%) & Dystrophin Immunostaining \\
\hline 1 & 5 & \multirow{2}{*}{44} & 65 & Positive \\
2 & 5 & & 70 & Positive \\
3 & 5 & & 43 & Positive \\
4 & 5 & $46-47$ & 30 & Positive \\
5 & 10 & & 40 & Positive \\
6 & 6 & $46-48$ & 40 & Positive \\
7 & 8 & $46-49$ & 65 & Positive \\
8 & 5 & & 51 & Positive \\
9 & 5 & & 100 & Positive \\
10 & 5 & $46-51$ & 100 & Positive \\
11 & 6 & & 82 & Negative \\
12 & 6 & $46-53$ & 65 & Positive \\
13 & 3 & & 45 & Negative \\
\hline
\end{tabular}

Myotubes were prepared from 13 DMD patients and transfected with $100 \mathrm{nM}$ of AO85. The percentages of exon-45-skipped mRNA among total mRNA were calculated as described in Figure 4. Dystrophin immunostaining was evaluated as described in Figure 5 and judged to be positive or negative. 


\section{Discussion}

Here, we have shown the process of developing the 2'-OMeRNA/ENA chimera AO against exon 45 and identified one suitable candidate, AO85. AO85 was effective in inducing exon 45 skipping and proved to be safe. These findings paved the way for clinical trials. In one clinical study, in fact, AO85 was shown safe and effective in increasing six-minute walk distance when it was injected intravenously at a dose of $0.5 \mathrm{mg} / \mathrm{kg} /$ week [38]. Currently, one $2^{\prime}$-OMeRNA/ENA chimera AO against dystrophin exon 45 (DS-5141b) is under clinical trial in Japan (JapicCTI No: 153072, http: //www.clinicaltrials.jp/user/cte_main.jsp). For induction of exon 45 skipping, another AO consisting of PMO (SRP-4045, Sarepta Therapeutics) is now in clinical trial (https:/ / clinicaltrials.gov).

Previous clinical trials of AO-mediated exon skipping have suggested a risk of renal impairment such as proteinuria [4]. Although single high-dose AO85 administration induced slight renal change, repeated administration of $\mathrm{AO} 85$ at the appropriate clinical dose $(0.5 \mathrm{mg} / \mathrm{kg})$ revealed no toxicity. AO85 was considered not toxic to the kidney.

Theoretically, exon 45 skipping efficiency should be equal in all affordable patients. However, our current investigation showed the surprising result of differences in exon skipping efficiency among the patients. Even in same-age patients, efficiency varied, suggesting that age is not a determining factor in efficacy differences. We also found a difference in exon skipping efficacy between patients with the $46-51$ deletion $(82 \%-100 \%)$ and those with the $46-47$ deletion $(30 \%-43 \%)$. This difference suggested a relation between the efficiency of exon skipping and the breakpoint sequence of the deletion mutation [39], which requires further study for confirmation.

In-frame exon 45-46 deletions result in a severe DMD phenotype [40]. Although these deletions are the outliers from the reading frame rule, the mechanism that produces the outlier is not known. Currently, the discrepancy between levels of exon skipping and dystrophin expression is not explainable. A limitation of this study was that quantitative evaluation was not performed, and more research is needed to clarify the mechanism that results in the discrepancy between exon skipping and dystrophin expression.

The cost of eteplirsen treatment was estimated $\$ 300,000-\$ 400,000$ in a year for one patient [22]. To reduce the cost, it is important to develop more efficient AO by either improving drug delivery [41] or using other modified nucleotides. In our case we employed ENA. In the current scheme the dose is only $0.5 \mathrm{mg} / \mathrm{kg}$ [37], while $30-50 \mathrm{mg} / \mathrm{kg}$ in eteplirsen [16]. Therefore, it is expected that the cost for treatment would be reduced using 2'-OMeRNA/ENA chimera. Efficient targeting of eteplirsen to cardiac muscle remains significant challenges [42]. Our result suggests that the delivery to cardiac muscle of 2'-OMeRNA/ENA chimera AO should be evaluated.

\section{Conclusions}

One 2'-OMeRNA/ENA chimera AO that induces exon 45 skipping was identified and shown to be non-toxic and effective. Although a discrepancy in levels between exon skipping and dystrophin expression was observed among samples, we expect that AO85 can be applied for AO-mediated exon skipping therapy as a fundamental treatment for DMD.

Acknowledgments: This work was supported in part by Translational Research Grant from NEDO, Intramural Research Grant for Neurological and Psychiatric Disorders of NCNP, Grant-in-Aid for Exploratory Research and Grant-in-Aid for Scientific Research from the Japan Society for the Promotion of Science, and Health and Labour Sciences Research Grant for Research on Psychiatric and Neurological Diseases and Mental Health.

Conflicts of Interest: M. Matsuo and Y. Takeshima are advisors for the Daiichi-Sankyo Co. Ltd., Tokyo. Other authors declare no conflict of interest.

\section{References}

1. Eagle, M.; Baudouin, S.V.; Chandler, C.; Giddings, D.R.; Bullock, R.; Bushby, K. Survival in Duchenne muscular dystrophy: Improvements in life expectancy since 1967 and the impact of home nocturnal ventilation. Neuromuscul. Disord. 2002, 12, 926-929. [CrossRef] 
2. Passamano, L.; Taglia, A.; Palladino, A.; Viggiano, E.; D'Ambrosio, P.; Scutifero, M.; Rosaria Cecio, M.; Torre, V.; DE Luca, F.; Picillo, E.; et al. Improvement of survival in Duchenne muscular dystrophy: Retrospective analysis of 835 patients. Acta Myol. 2012, 31, 121-125. [PubMed]

3. Van Deutekom, J.C.; van Ommen, G.J. Advances in Duchenne muscular dystrophy gene therapy. Nat. Rev. Genet. 2003, 4, 774-783. [CrossRef] [PubMed]

4. Robinson-Hamm, J.N.; Gersbach, C.A. Gene therapies that restore dystrophin expression for the treatment of Duchenne muscular dystrophy. Hum. Genet. 2016, 135, 1029-1040. [CrossRef] [PubMed]

5. Takeshima, Y.; Nishio, H.; Sakamoto, H.; Nakamura, H.; Matsuo, M. Modulation of in vitro splicing of the upstream intron by modifying an intra-exon sequence which is deleted from the dystrophin gene in dystrophin kobe. J. Clin. Investig. 1995, 95, 515-520. [CrossRef] [PubMed]

6. Monaco, A.P.; Bertelson, C.J.; Liechti-Gallati, S.; Moser, H.; Kunkel, L.M. An explanation for the phenotypic differences between patients bearing partial deletions of the DMD locus. Genomics 1988, 2, 90-95. [CrossRef]

7. Matsuo, M. Duchenne/becker muscular dystrophy: From molecular diagnosis to gene therapy. Brain Dev. 1996, 18, 167-172. [CrossRef]

8. Takeshima, Y.; Yagi, M.; Wada, H.; Ishibashi, K.; Nishiyama, A.; Kakumoto, M.; Sakaeda, T.; Saura, R.; Okumura, K.; Matsuo, M. Intravenous infusion of an antisense oligonucleotide results in exon skipping in muscle dystrophin mRNA of Duchenne muscular dystrophy. Pediatr. Res. 2006, 59, 690-694. [CrossRef] [PubMed]

9. Matsuo, M.; Takeshima, Y.; Nishio, H. Contributions of Japanese patients to development of antisense therapy for DMD. Brain Dev. 2016, 38, 4-9. [CrossRef] [PubMed]

10. Wilton, S.D.; Veedu, R.N.; Fletcher, S. The emperor's new dystrophin: Finding sense in the noise. Trends Mol. Med. 2015, 21, 417-426. [CrossRef] [PubMed]

11. Le, B.T.; Veedu, R.N.; Fletcher, S.; Wilton, S.D. Antisense oligonucleotide development for the treatment of muscular dystrophies. Expert Opin. Orph. Drugs 2016, 4, 139-152.

12. Chen, S.; Le, B.T.; Rahimizadeh, K.; Shaikh, K.; Mohal, N.; Veedu, R.N. Synthesis of a morpholino nucleic acid(MNA)-uridine phosphoramidite, and exon skipping using MNA/20-O-Methyl mixmer antisense oligonucleotide. Molecules 2016. [CrossRef] [PubMed]

13. Le, B.T.; Chen, S.; Abramov, M.; Herdewijn, P.; Veedu, R.N. Evaluation of anhydrohexitol nucleic acid, cyclohexenyl nucleic acid and d-altritol nucleic acid-modified 2'-O-methyl RNA mixmer antisense oligonucleotides for exon skipping in vitro. Chem. Commun. 2016, 52, 13467-13470. [CrossRef] [PubMed]

14. Kinali, M.; Arechavala-Gomeza, V.; Feng, L.; Cirak, S.; Hunt, D.; Adkin, C.; Guglieri, M.; Ashton, E.; Abbs, S.; Nihoyannopoulos, P.; et al. Local restoration of dystrophin expression with the morpholino oligomer AVI-4658 in Duchenne muscular dystrophy: A single-blind, placebo-controlled, dose-escalation, proof-of-concept study. Lancet Neurol. 2009, 8, 918-928. [CrossRef]

15. Goemans, N.M.; Tulinius, M.; van den Akker, J.T.; Burm, B.E.; Ekhart, P.F.; Heuvelmans, N.; Holling, T.; Janson, A.A.; Platenburg, G.J.; Sipkens, J.A.; et al. Systemic administration of PRO051 in Duchenne's muscular dystrophy. N. Engl. J. Med. 2011, 364, 1513-1522. [CrossRef] [PubMed]

16. Mendell, J.R.; Rodino-Klapac, L.R.; Sahenk, Z.; Roush, K.; Bird, L.; Lowes, L.P.; Alfano, L.; Gomez, A.M.; Lewis, S.; Kota, J.; et al. Eteplirsen for the treatment of Duchenne muscular dystrophy. Ann. Neurol. 2013, 74, 637-647. [CrossRef] [PubMed]

17. Voit, T.; Topaloglu, H.; Straub, V.; Muntoni, F.; Deconinck, N.; Campion, G.; De Kimpe, S.J.; Eagle, M.; Guglieri, M.; Hood, S.; et al. Safety and efficacy of drisapersen for the treatment of Duchenne muscular dystrophy (DEMAND II): An exploratory, randomised, placebo-controlled phase 2 study. Lancet Neurol. 2014, 13, 987-996. [CrossRef]

18. Mendell, J.R.; Goemans, N.; Lowes, L.P.; Alfano, L.N.; Berry, K.; Shao, J.; Kaye, E.M.; Mercuri, E. Longitudinal effect of eteplirsen versus historical control on ambulation in Duchenne muscular dystrophy. Ann. Neurol. 2016, 79, 257-271. [CrossRef] [PubMed]

19. Goemans, N.M.; Tulinius, M.; van den Hauwe, M.; Kroksmark, A.K.; Buyse, G.; Wilson, R.J.; van Deutekom, J.C.; de Kimpe, S.J.; Lourbakos, A.; Campion, G. Long-term efficacy, safety, and pharmacokinetics of drisapersen in Duchenne muscular dystrophy: Results from an open-label extension study. PLoS ONE 2016, 11, e0161955. 
20. Aartsma-Rus, A.; Ferlini, A.; Goemans, N.; Pasmooij, A.M.; Wells, D.J.; Bushby, K.; Vroom, E.; Balabanov, P. Translational and regulatory challenges for exon skipping therapies. Hum. Gene Ther. 2014, 25, 885-892. [CrossRef] [PubMed]

21. FDA grants accelerated approval to first drug for Duchenne muscular dystrophy. Avaliable online: http://www.fda.gov/NewsEvents/Newsroom/PressAnnouncements/ucm521263.htm (accessed on 19 September 2016).

22. Aartsma-Rus, A.; Krieg, A.M. FDA approves eteplirsen for Duchenne muscular dystrophy: The next chapter in the eteplirsen saga. Nucleic Acid Ther. 2016. [CrossRef] [PubMed]

23. Unger, E.F.; Califf, R.M. Regarding eteplirsen for the treatment of Duchenne muscular dystrophy. Ann. Neurol. 2016, 81, 162-164. [CrossRef] [PubMed]

24. Mendell, J.R. Eteplirsen improves function and partially restores dystrophin. Ann. Neurol. 2016. [CrossRef]

25. Stein, C.A. Eteplirsen approved for Duchenne muscular dystrophy: The FDA faces a difficult choice. Mol. Ther. 2016, 24, 1884-1885. [CrossRef] [PubMed]

26. Toh, Z.Y.; Thandar Aung-Htut, M.; Pinniger, G.; Adams, A.M.; Krishnaswarmy, S.; Wong, B.L.; Fletcher, S.; Wilton, S.D. Deletion of dystrophin in-frame exon 5 leads to a severe phenotype: Guidance for exon skipping strategies. PLOS ONE 2016, 11, e0145620. [CrossRef] [PubMed]

27. Koizumi, M. ENA oligonucleotides as therapeutics. Curr. Opin. Mol. Ther. 2006, 8, 144-149. [PubMed]

28. Morita, K.; Hasegawa, C.; Kaneko, M.; Tsutsumi, S.; Sone, J.; Ishikawa, T.; Imanishi, T.; Koizumi, M. 2'-O,4'-C-ethylene-bridged nucleic acids (ENA) with nuclease-resistance and high affinity for RNA. Nucleic Acids Res. Suppl. 2001, 241-242. [CrossRef]

29. Morita, K.; Hasegawa, C.; Kaneko, M.; Tsutsumi, S.; Sone, J.; Ishikawa, T.; Imanishi, T.; Koizumi, M. 2'-O,4'-C-ethylene-bridged nucleic acids (ENA): Highly nuclease-resistant and thermodynamically stable oligonucleotides for antisense drug. Bioorg. Med. Chem. Lett. 2002, 12, 73-76. [CrossRef]

30. Morita, K.; Takagi, M.; Hasegawa, C.; Kaneko, M.; Tsutsumi, S.; Sone, J.; Ishikawa, T.; Imanishi, T.; Koizumi, M. Synthesis and properties of 2'-O,4'-C-ethylene-bridged nucleic acids (ENA) as effective antisense oligonucleotides. Bioorg. Med. Chem. 2003, 11, 2211-2226. [CrossRef]

31. Koizumi, M. 2'-O, 4'-C-ethylene-bridged nucleic acids (ENA) as next-generation antisense and antigene agents. Biol. Pharm. Bull. 2004, 27, 453-456. [CrossRef] [PubMed]

32. Yagi, M.; Takeshima, Y.; Suruno, A.; Takagi, M.; Koizumi, M.; Matsuo, M. Chimeric RNA and 2'-O, $4^{\prime}$-C-ethylene-bridged nucleic acids have stronger activity than phosphorothioate oligodeoxynucleotides in induction of exon-19 skipping in dystropin mRNA. Oligonucleotides 2004, 14, 33-40. [CrossRef] [PubMed]

33. Surono, A.; Tran, V.K.; Takshima, Y.; Wada, H.; Yagi, M.; Takagi, M.; Koizumi, M.; Matsuo, M. Chimeric RNA/ethylene bridged nucleic acids promote dystrophin expression in myocytes of Duchenne muscular dystrophy by inducing skipping of the nonsense-mutation-encoding exon. Hum. Gene Ther. 2004, 15, 749-757. [CrossRef] [PubMed]

34. Takeshima, Y.; Yagi, M.; Okizuka, Y.; Awano, H.; Zhang, Z.; Yamauchi, Y.; Nishio, H.; Matsuo, M. Mutation spectrum of the dystrophin gene in 442 duchenne/becker muscular dystrophy cases from one Japanese referral center. J. Hum. Genet. 2010, 55, 379-388. [CrossRef] [PubMed]

35. Takeshima, T.; Yagi, M.; Matsuo, M. Optimizing RNA/ENA chimeric antisense oligonucleotides using in vitro splicing. In Exon Skipping: Methods and Protocols; Aartsma-Rus, A., Ed.; Human Press: New York, NY, USA, 2012; Volume 867, pp. 131-141.

36. Malueka, R.; Yagi, M.; Awano, H.; Lee, T.; Dwianingsih, E.K.; Nishida, A.; Takeshima, Y.; Matsuo, M. Antisense oligonucleotide induced dystrophin exon 45 skipping at a low half-maximal effective concentration in a cell-free splicing system. Nucleic Acid Ther. 2011, 21, 347-353. [CrossRef] [PubMed]

37. Malueka, R.G.; Dwianingsih, E.K.; Yagi, M.; Lee, T.; Nishida, A.; Iijima, K.; Takeshima, Y.; Matsuo, M. Phosphorothioate modification of chimeric 2'-O-methyl RNA/ethylene-bridged nucleic acid oligonucleotides increases dystrophin exon 45 skipping capability and reduces cytotoxicity. Kobe J. Med. Sci. 2015, 60, E86-E94. [PubMed]

38. Takeshima, Y.; Lee, T.; Shimomura, H.; Tanaka, Y.; Awano, H.; Nishida, A.; Ojima, I.; Minami, S.; Nakagawa, A.; Iijima, K.; et al. A new antisense oligonucleotide composed of RNA/ENA chimera (AO85) against dystrophin exon 45 significantly increased six-minute walk distance in Duchenne muscular dystrophy. (Abstract/Program \#P08-111). In Presented at the 5th International Congress of Myology, Lyon, France, 14-18 March 2016. 
39. Dwianingsih, E.K.; Malueka, R.G.; Nishida, A.; Itoh, K.; Lee, T.; Yagi, M.; Iijima, K.; Takeshima, Y.; Matsuo, M. A novel splicing silencer generated by dmd exon 45 deletion junction could explain upstream exon 44 skipping that modifies dystrophinopathy. J. Hum. Genet. 2014, 59, 423-429. [CrossRef] [PubMed]

40. Findlay, A.R.; Wein, N.; Kaminoh, Y.; Taylor, L.E.; Dunn, D.M.; Mendell, J.R.; King, W.M.; Pestronk, A.; Florence, J.M.; Mathews, K.D.; et al. Clinical phenotypes as predictors of the outcome of skipping around dmd exon 45. Ann. Neurol. 2015, 77, 668-674. [CrossRef] [PubMed]

41. Kendall, G.C.; Mokhonova, E.I.; Moran, M.; Sejbuk, N.E.; Wang, D.W.; Silva, O.; Wang, R.T.; Martinez, L.; Lu, Q.L.; Damoiseaux, R.; et al. Dantrolene enhances antisense-mediated exon skipping in human and mouse models of Duchenne muscular dystrophy. Sci. Transl. Med. 2012. [CrossRef] [PubMed]

42. Arechavala-Gomeza, V.; Anthony, K.; Morgan, J.; Muntoni, F. Antisense oligonucleotide-mediated exon skipping for Duchenne muscular dystrophy: Progress and challenges. Curr. Gene Ther. 2012, 12, $152-160$. [CrossRef] [PubMed]

(C) 2017 by the authors; licensee MDPI, Basel, Switzerland. This article is an open access article distributed under the terms and conditions of the Creative Commons Attribution (CC BY) license (http:/ / creativecommons.org/licenses/by/4.0/). 